\title{
« On a beaucoup à dire et peu à raconter »
}

Correspondance entre Romain Rolland et Frédéric Ferrière 1914-1924

\section{Martine Ruchat}

\section{(2) OpenEdition}

\section{Journals}

Édition électronique

URL : http://journals.openedition.org/edl/347

DOI : 10.4000/edl.347

ISSN : 2296-5084

Éditeur

Université de Lausanne

Édition imprimée

Date de publication : 15 septembre 2012

Pagination : 143-172

ISBN : 978-2-940331-28-4

ISSN : 0014-2026

Référence électronique

Martine Ruchat, « « On a beaucoup à dire et peu à raconter » », Études de lettres [En ligne], 3 | 2012, mis en ligne le 15 septembre 2015, consulté le 19 décembre 2020. URL : http://journals.openedition.org/ edl/347 ; DOI : https://doi.org/10.4000/edl.347 


\section{"ON A BEAUCOUP À DIRE ET PEU À RACONTER» ${ }^{1}$ CORRESPONDANCE ENTRE ROMAIN ROLLAND ET FRÉDÉRIC FERRIÈRE 1914-1924}

Cet article, s'appuyant sur la correspondance entre Romain Rolland et le médecin genevois Frédéric Ferrière, reprend sous un angle original la période où Rolland est en Suisse, et particulièrement à Genève, engagé à la section civile de l'Agence des prisonniers de guerre, dont l'objectif est d'informer les familles sur les conditions et l'état de santé des prisonniers. L'auteure souligne en particulier, grâce à leurs échanges épistolaires, la relation d'amitié et le soutien réciproque dans leurs engagements sociaux et politiques. C'est en particulier le rôle de Ferrière comme médecin et ami intime de Rolland, et plus largement celui d'un combattant pour la justice et pour une haute idée de l'humanité, que l'on découvre. Il restera fidèle à Rolland après son départ de l'Agence, en 1915, un signe aussi de son engagement affectif et politique. Grâce à cette étude, c'est tout un réseau cosmopolite qui se dessine autour de la figure centrale de Ferrière sur fond de guerre, de révolution bolchevique et de tensions politiques. A travers ce récit, construit essentiellement sur l'archive épistolaire, se profile aussi l'image d'un Romain Rolland attachant et soucieux d'aide humanitaire (pour les prisonniers et leurs familles, pour les enfants de Vienne) et très attaché à Frédéric Ferrière (première visite, première lettre sitôt arrivé à Genève), ce qui explique en partie l'importante correspondance familiale qui se poursuivra avec ses descendants Maya, Adolphe et Frédéric Ferrière fils (424 lettres conservées jusqu’à ce jour).

I. Lettre de Romain Rolland à Frédéric Ferrière, 7 mai 1924. 


\section{L'enracinement d'une longue amitié}

En été 1914, Romain Rolland est à Vevey. Son père, Emile, et sa sœur, Madeleine, sont à Schönbrunn, dans une station climatique au bord du lac de Zoug. Sa mère, Antoinette-Marie, est restée dans leur maison familiale de Clamecy. Par une lettre du 26 juillet, il la prie de venir le rejoindre, par crainte d'une déclaration de la guerre. Rolland retourne à Paris un seul jour, le 29 juillet, pour «mettre ses affaires en sûreté et ramener quelques titres " ${ }^{2}$. Jean Jaurès est assassiné le 31 juillet et l'Allemagne déclare la guerre à la Russie le $1^{\text {er }}$ août. Ce même jour, la mère de Rolland rejoint son fils à Vevey et Madeleine les retrouve à la fin du mois. Ainsi commence pour ces années de guerre une vie de famille chaotique dans laquelle le médecin genevois Frédéric Ferrière (1848-1924), vice-président de la Croix-Rouge, chef du service sanitaire de l'Agence des prisonniers de guerre et fondateur de sa section civile, jouera un rôle essentiel ${ }^{3}$.

Les liens de Romain Rolland avec la Suisse sont déjà bien connus. Dans sa thèse, Claire Basquin évoque à plusieurs reprises ses vacances en Suisse où il se rend chaque été depuis l'âge de 20 ans $^{4}$. Bernard Duchatelet, dans sa biographie qui repose essentiellement sur le Journal de Rolland et sur ses écrits, évoque de même certains éléments ${ }^{5}$. Antoinette Blum, quant à elle, a édité la correspondance entre Rolland et Charles Baudouin, français, établi à Genève depuis l'automne $1915^{6}$. Or, les liens épistolaires entretenus avec Ferrière, commencés lors de ses quelques mois passés à la section civile de l'Agence des prisonniers de guerre, à Genève, puis ceux développés entre les deux familles, sont encore mal connus ${ }^{7}$. p. 86.

2. C. Basquin, Romain Roland et l'Agence des prisonniers de Genève (1914-1916),

3. Voir R. Armanios, Le Dr. Ferrière; A. Ferrière, Le Dr. Frédéric Ferrière; G. Werner, «Frédéric Ferrière 1848-1924».

4. Ibid.

5. B. Duchatelet, Romain Rolland tel qu'en lui-même. Concernant son travail à l'Agence des prisonniers de guerre, voir notamment le chapitre 4, "Au-dessus de la mêlée".

6. A. Blum (éd.), Correspondance entre Romain Rolland et Charles Baudouin.

7. Romain Rolland introduit ainsi, dans son journal, Frédéric Ferrière: «La famille Ferrière était originaire de Normandie, mais établie à Genève depuis le début du XVIII ${ }^{\mathrm{e}}$ siècle. Frédéric Ferrière, né à Genève en 1848, docteur d'Heidelberg, travaille 
La relation épistolaire entre Romain Rolland et la famille Ferrière a déjà été présentée partiellement par deux historiens: en 1963 par Sven Stelling Michaud et, en 1985, par Marc Reinhardt ${ }^{8}$. Le premier offre à lire dix-sept lettres entre Romain Rolland et le pédagogue Adolphe Ferrière, fils du médecin. Quant à Reinhardt, il semble avoir connaissance d'une importante correspondance, puisqu'il parle des "douzaines et des douzaines de lettres sur une période de trente ans». Or il n'offre des extraits que de quarante d'entre elles dont une seule de Frédéric Ferrière à Romain Rolland ${ }^{9}$. Bien que ces lettres soient connues d'un public averti, la donation récente de celles-ci aux Archives d'Etat de Genève facilite leur accès et stimule désormais la recherche ${ }^{10}$. Les lettres de la famille Ferrière conservées à la Bibliothèque nationale de Paris complètent ce fonds genevois assurant le croisement (non exhaustif). En 1963, Stelling Michaud n'a en main que trois lettres d'Adolphe Ferrière à Rolland, il y en a aujourd'hui trente-deux. Quant aux lettres du médecin à Rolland, elles sont actuellement au nombre de trente-neuf. Ainsi, même si des extraits de ces lettres ont pu prendre place dans ces deux

dans les ambulances pendant la guerre de 1870. En 1876, fonde la Croix-Rouge au Monténégro, dirige en 1877 une ambulance monténégrine, pendant la guerre RussoTurque. Entre au comité International de la Croix-Rouge en 1884. L'oriente vers les œuvres de paix. Présente en 1907 à la conférence de Londres un mémoire sur la "Participation de la Croix-Rouge à la lutte contre la tuberculose". Fait de nombreux voyages en Serbie, Monténégro, Bulgarie, Grèce. En août 1914, il fonde à l'Agence des prisonniers de guerre une section spéciale des civils. Mission en Allemagne en 1915. Mission dans les Balkans en 1919. Il y sacrifie tous ses autres travaux et ses intérêts personnels. Il prend une part importante à la fondation de l'Union Internationale de secours aux enfants (IUSE). Nombreuses et longues missions à Vienne, où il a centralisé les diverses œuvres d'assistance des Croix-Rouges, et où il prend une part active à l'organisation de la lutte contre le typhus.» Extrait du Journal $1^{\text {er }}$ janvier-6 décembre 1924. Lettre de Romain Rolland à M $^{\text {me }}$ Frédéric Ferrière, samedi 14 juin 1924, Archives d'état de Genève.

8. P. Abraham et al., Romain Rolland et M. Reinhardt, "Romain Rolland et les Ferrière».

9. Vingt-quatre lettres de Romain Rolland à Frédéric Ferrière, neuf lettres à Maya Ferrière (une lettre d'elle à Rolland); entre Frédéric Ferrière (fils) et Romain Rolland (six lettres), Isabelle Ferrière et Romain Rolland (trois lettres), la même à la mère de Romain Rolland (une lettre).

Io. Correspondance dont la photocopie avait d'ailleurs été déposée par Claude Ferrière, fils d'Adolphe Ferrière, à la Bibliothèque nationale de France. 
articles, l'ensemble des lettres n'a jamais été publié et analysé ${ }^{11}$. Cette correspondance familiale fera l'objet d'une publication ultérieure ${ }^{12}$.

Dans cet article, je me centrerai sur la période où s'établissent les liens de Romain Rolland avec la famille Ferrière, et en particulier avec Frédéric Ferrière, afin de mettre en lumière non seulement le rôle de Rolland dans l'Agence, rôle déjà étudié par Claire Basquin, mais surtout celui de soutien de famille qu'a été Frédéric Ferrière dans cette période difficile traversée par Rolland pendant son séjour à Genève, entre 1914 et 1915, et même au-delà jusqu'à la mort du médecin en 1924. Frédéric Ferrière sera notamment son conseiller en matière de santé, ainsi que pour celle de sa mère. Pendant son travail à l'Agence, les lettres ne disent pas tout, car elles peuvent être lues. Bien des questions seront discutées de vive voix ne laissant ainsi aucune trace épistolaire. Cette correspondance montre aussi par ses rythmes et interruptions les difficultés de la vie quotidienne en période de guerre (fermeture des frontières, rationnement, blocage des échanges postaux).

Ce moment fondateur de leur amitié qu'est l'Agence ne cesse de les réunir même au-delà des mois effectifs de collaboration à la section civile, non seulement parce que Romain Rolland continue de recevoir des demandes personnelles (par des parents de prisonniers) qui ne passent pas par la Croix-Rouge (et qu'il doit renvoyer à Ferrière ou à sa fille Maya qui y travaille aussi), mais aussi parce que pendant cette période une amitié et une complicité intellectuelle se sont enracinées, lesquelles se transmettront d'une génération à l'autre pendant près d'un demisiècle. L'Agence ferme ses portes en mars 1919 non sans avoir mis en mouvement tout un microcosme cosmopolite.

\section{Une correspondance familiale}

Dès la première lettre conservée, en date du 23 octobre 1914, le ton est donné entre les deux principaux protagonistes, amitié, admiration

II. Je remercie Patricia-Laure Pasche pour avoir effectué la retranscription de l'ensemble des lettres de cette correspondance familiale.

I2. L'Association Romain Rolland a, grâce à la publication de nombreux Cahiers (vingt-neuf jusqu'à ce jour), commencé à montrer les diverses facettes et l'étendue du réseau de ses correspondantes et correspondants qui participe, comme toute correspondance, à reconstruire sa biographie. 
et dévouement, et les autres personnages de la famille peuvent successivement entrer en scène. Madeleine Rolland qui écrit à Adolphine Ferrière-Faber, la femme de Frédéric Ferrière, le 12 mai 1915, lui envoyant une circulaire l'invitant à rejoindre une réunion d'un comité directeur provisoire et d'un secrétariat de l'«Alliance d'éducation sociale et civique" introduisant un intérêt entre les deux familles pour cette «Reconstruction de la Civilisation Européenne sur un véritable plan de Liberté et de Fraternité» ${ }^{13}$. Puis le 20 mai 1915, Adolphe Ferrière, un des trois fils de Frédéric Ferrière envoie, en hommage à Rolland, sa thèse de doctorat, conçue il y a onze ans, et qu'il soutiendra le jeudi 27 mai. Sourd partiellement depuis l'âge de 14 ans, puis totalement depuis l'âge de 42 ans (en 1921), Ferrière regrette de ne pouvoir parler avec Rolland. Il lui écrit: "Voici ma thèse. Veuillez l'accepter comme un hommage d'un homme qui ne peut vous entendre, mais qui peut vous lire et vous a lu avec enthousiasme. Votre sincérité malgré tout, votre perspicacité psychologique, voilà deux qualités auxquelles j'ambitionnerais d'atteindre au même degré que vous. Mais je me rends compte que mon ouïe est un obstacle à la seconde en m'empêchant de connaître le monde, les gens et les choses comme vous avez pu les connaître» (lettre du 20 mai 1915) ${ }^{14}$. Il l'invite à venir prendre le thé dans son chalet de Blonay s'il fait un séjour dans la région ${ }^{15}$. Rolland lit sa thèse avec un grand intérêt et il la cite dans son article «Le meurtre des élites" du Journal de Genève (lettre du 14 juin 1915).

Le 6 septembre de cette même année, la mère de Rolland écrit à Adolphine Ferrière-Faber, une lettre dans laquelle elle lui fait part de son envie de faire connaître Adolphe, et sa femme Isabelle, à sa fille Madeleine. Maya Ferrière écrit une première lettre, le 29 janvier 1916, à Romain Rolland accompagnant "quelques fleurettes" pour ses 50 ans, à laquelle il répond le jour même. La mère de Rolland écrit aussi à Maya le 21 juin 1916. Et Romain Rolland écrit à Adolphine Ferrière-Faber le 28 mars 1917. Les lettres d'Isabelle Ferrière à Romain Rolland, élargissent le réseau des croisements interfamiliaux le 22 mai 1919, puis

I3. La lettre est signée Henri Oger, administrateur des Services Civils de l'IndeChine.

I4. Lettre retranscrite par Sven Stelling Michaud.

I5. Plus tard lorsqu'ils se rencontreront, les mots de Romain Rolland seront écrits pour Adolphe de la main de sa femme Isabelle (voir par exemple 3 septembre 1924, Archives d'Etat de Genève, Archives privées 379). 
c'est Madeleine qui écrira à Frédéric Ferrière le 31 mai 1919 et le père de Romain Rolland, Emile, écrit à Maya le 22 février 1921; Madeleine écrit aussi à Maya le 3 octobre 1921 pour avoir des nouvelles de "notre cher malade» ${ }^{16}$. Madeleine envoie une lettre à Adolphe et Isabelle le 11 juillet 1921. Emile Rolland entre en relation épistolaire avec Adolphe Ferrière le 15 avril 1924. Et Frédéric Ferrière (fils) entre dans la ronde en écrivant à Romain Rolland dès le 28 janvier 1924, lettres auxquelles répond Romain Rolland (lettres conservées dès le 28 janvier 1936). Maya et Isabelle Ferrière écrivent aussi à $\mathrm{M}^{\text {me }}$ Rolland (respectivement le 13 janvier 1935 et le 10 mars 1935). Enfin Marie Rolland, la femme de Romain, dite Macha, écrit à Adolphe Ferrière le 7 décembre 1945 et Adolphe écrit une lettre à la mère de Romain Rolland le 3 août 1950 (une seule lettre est conservée). Une lettre de Macha à Maya clôt ces échanges épistolaires le 31 janvier 1962. Il y a donc aujourd'hui un ensemble de 424 lettres entre onze épistoliers dont 238 de Romain Rolland, entre le 23 octobre 1914 et le 31 janvier 1962, ce qui nous permet d'avoir une vision plus précise de leurs échanges ${ }^{17}$.

\section{Un réseau épistolaire}

D'autres personnages apparaissent dans cette correspondance qui font partie de ce qu'on appelle aujourd'hui leur «réseau » : à commencer par Henri Guilbeaux ${ }^{18}$ qui est présenté dès la troisième lettre de Romain Rolland à Ferrière comme un ami, désireux de travailler à l'Agence, un "garçon loyal, actif et débrouillard, habitué au travail de bureau (il a été secrétaire de Jean Finot à la Revue des revues), connaissant l'allemand et ayant conservé dans cette guerre un esprit assez impartial» (lettre du 22 mai 1915). Ce qu'il fera, comme chef de bureau, pour 250 francs par

I6. Frédéric Ferrière a un cancer de l'intestin qui sera traité par des rayons au radium, puis opéré en octobre.

17. Je remercie bien sincèrement Madame Marie-Laure Prévost, archiviste à la Bibliothèque nationale de France, pour avoir mis à ma disposition la correspondance Ferrière du fonds Romain Rolland.

I8. Henri Guilbeaux (1885-1938) : écrivain, journaliste, militant socialiste et pacifiste, deviendra communiste (trotskyste). 
mois dès le $1^{\text {er }}$ juin. Il y a Paul Seippel ${ }^{19}$ que le Frédéric Ferrière doit soigner pour une crise de colique hépatique alors qu'ils sont tous deux en Valais; Stefan Zweig ${ }^{20}$ qu'il doit saluer pour Rolland à Vienne où le médecin se rend en août 1915: "Stefan Zweig (VIII Kochgasse 8), veuillez lui transmettre mes affectueux souvenirs. C'est un cœur généreux et un esprit vraiment européen. Il sait beaucoup de choses de la situation actuelle, et je crois que vous pourrez avoir intérêt à causer avec lui. Il vous connaît et vous respecte; et je suis sûr qu'il se mettrait tout à votre disposition, autant que lui permettront les exigences de son service: car il doit être occupé, au ministère de la guerre" (lettre du 31 août 1915). Puis c'est la demande d'un conseil pour une pension où pourrait être envoyé son ami Pierre Jean Jouve ${ }^{21}$ qui est, lui aussi, malade: "Il a passé l'année dernière à soigner les malades dans un hôpital de province, section des contagieux, et il y a attrapé coup sur coup rougeole, scarlatine et coqueluche. Déjà délicat de santé, il doit être assez sérieusement atteint. Il demande où il pourrait aller de préférence. Il lui faudrait un lieu abrité, 1200 à 1500 m» (lettre du 4 octobre 1915); Maurice Pottecher, écrivain français présenté comme un «excellent ami» ${ }^{22}$. Paul Amann, professeur de littérature française, ami de Stefan Zweig, écrivain de grand talent (selon Romain Rolland) auquel Frédéric Ferrière doit transmettre son souvenir ${ }^{23}$. Et Nicolaï Roubakine qui est parti pour quelques jours en Allemagne et n'est plus autorisé à rentrer dans son domicile de Clamecy (lettre du 27 août 1919) ${ }^{24}$. Il y aura aussi son amie

19. Paul Seippel (1858-1926) : professeur de littérature française à l'Ecole polytechnique fédérale de Zurich et critique littéraire au Journal de Genève, premier biographe de Romain Rolland, Romain Rolland. L'homme et l'ouvre, Paris, Ollendorf, 1913; il est à la base d'un texte d'appel à l'union lancé par 300 professeurs d'universités suisses au mois de mai 1915.

20. Stefan Zweig (1881-1942) : écrivain (romancier, essayiste, nouvelliste, dramaturge, traducteur) autrichien; docteur en philosophie à 23 ans, il se distingue alors par sa grande production littéraire et épistolaire, ses nombreux voyages, ses prises de position politique.

2I. Pierre Jean Jouve (1887-1976) : poète et romancier, français, pacifiste internationaliste, installé en Suisse depuis 1915, proche de Zweig; il publie dans Le Carmel, dans la revue Demain et dans Les Tablettes.

22. Maurice Pottecher (1867-1960) : écrivain et homme de théâtre, fondateur du théâtre du Peuple à Bussang dans les Vosges, en 1895.

23. Paul Amann (1884-1958): écrivain et traducteur autrichien.

24. Nicolaï Alexandrovitch Roubakine (1862-1946): savant russe, fondateur et théoricien de la psychologie bibliologique; il crée un Institut de psychobibliologie. Exilé 
de Zurich Elsa Hartoch (russe naturalisée suisse travaillant, elle aussi, à l'Agence) et ses amis de Vienne dont il donne la liste à Maya Ferrière dans une lettre du 29 janvier 1921 : $D^{r}$ Alice Furtmüller, $M^{\text {me }}$ Dr. phil. Anna Nussbaum, Valère de Ferenczy.

A travers cette correspondance, c'est aussi tout un monde qui se dessine: le monde de ceux qui, comme le dit Rolland de Guilbeaux, ont "conservé dans cette guerre un esprit assez impartial» (lettre du 22 mai $1915^{25}$ ) et qui ont un esprit comme celui de Frédéric Ferrière que Rolland décrit dans ses lettres: «[...] large esprit de justice et d'humanité, à la fois sensible à toutes les iniquités et indulgent à toutes les faiblesses de cette pauvre espèce humaine" (lettre du 22 mai 1915) et comme Frédéric Ferrière le relève pour Romain Rolland: habité par un «besoin de droiture, de justice et de générosité» (lettre du 5 juillet 1915).

Esprit qu'ils ont probablement en commun.

\section{Une correspondance à l'abri de la mêlée}

C'est donc le 15 août 1914 que la Croix-Rouge internationale décide, par mesure urgente, d'ouvrir dans le Palais Hainard, à Genève, une Agence centrale des prisonniers de guerre dont l'objectif est de rétablir les liens familiaux entre les personnes séparées par la guerre, et ce «dans le cas des prisonniers de guerre, des internés civils et des civils des régions occupées ${ }^{26}$. Ce sera chose faite le 21 août 1914. Quant à la section civile, elle est créée par Ferrière le 21 septembre. Elle est logée, dès le 12 octobre, au Musée Rath avec l'Agence qui y déménage. Ce bureau civil est un peu en marge de l'Agence, les prisonniers civils n'ayant pas été prévus par la Convention de La Haye.

Si les dates d'installation de Romain Rolland à Genève et dans son travail à l'Agence varient selon les auteurs, une page du Journal des

en Suisse en 1907, il prend contact avec l'Institut J.-J. Rousseau, en particulier avec Adolphe Ferrière, Edouard Claparède et Pierre Bovet. Le 20 octobre 1916, il y fonde la section de bibliopsychologie qu'il dirigera. Il publie à Paris chez J. Povolozky en 1922, Introduction à la psychologie bibliologique. Théorie et pratique.

25. Lettre retranscrite en partie par Marc Reinhardt.

26. L'agence internationale des prisonniers de guerre, p. 3. Il y aurait, selon une lettre de Frédéric Ferrière, 30000 à 40000 internés en Suisse en décembre 1917 (lettre du 20 décembre 1917). 
années de guerre laisse pourtant clairement comprendre qu'il a écrit au président de la Croix-Rouge, Gustave Ador, le 20 septembre, et qu'il a reçu une réponse favorable le 24 septembre ${ }^{27}$; qu'il y arrive le 6 octobre et commence une heure après son arrivée ${ }^{28}$. Rolland quitte après dix mois la section civile, le 3 juillet 1915. Il semblerait d'ailleurs que ce soit Madeleine Rolland qui, la première, ait contacté l'Agence. Elle y travaille du 19 septembre au 22 octobre, date à laquelle elle regagne Paris.

Rolland prend place dans cette organisation de la section civile de l'Agence des prisonniers de guerre, dans laquelle une quarantaine de personnes trient environ 1200 lettres par jour, les classent par villes et pays et font un résumé des informations demandées sur une «carte d'enquête». "Ces pauvres lettres s'accumulent et cela nous fait mal de songer à toutes les inquiétudes mortelles que cela représente. La majorité de ces lettres sont adressées à des épouses et des mères" écrit dans son rapport de fin d'année 1915 Hélène Appia. Elle ajoute: «Beaucoup d'étrangers sont venus s'installer à Genève pour être à proximité de l'Agence et par là même plus à portée des communications avec les prisonniers qui les intéressent. ${ }^{29}$.

Rolland se rend à l'Agence les après-midi; il lit les lettres, remplit les fiches, vérifie les informations, contacte les états-majors des camps d'internement. Mais il est plus qu'un simple collaborateur parmi les cent vingt-trois bénévoles que l'Agence a recrutés. Ferrière compte sur lui pour écrire des articles, convaincu qu'il est «que l'action sur les consciences fait plus que les démarches officielles" (lettre du 23 octobre 1914) et que mettre sa signature aura un effet dans les démarches faites: «[...] il va sans dire que si votre nom figure en bas d'une démarche de ce fait cela aura plus de poids que s'il y figurait un nom moins autorisé

27. Deux lettres de même le confirment: l'une écrite à son amie Louise Cruppi, le 24 septembre, l'autre à Georges Wagnière directeur du Journal de Genève, le 29 septembre; cf. C. Basquin, Romain Rolland et l'Agence des prisonniers de Genève (19141916), p. 98.

28. Basquin parle des 2 et 6 octobre et du 24 septembre (ibid., p. 97 sq.); Stelling Michaud donne celle d'août 1914 (in P. Abraham et al., Romain Rolland, p. 185); Duchatelet parle des "premiers jours d'octobre" (Romain Rolland tel qu'en lui-même, p. 174); Marc Reinhardt écrit "au début de la guerre de 1914 ", soit début août («Romain Rolland et les Ferrière», p. 137).

29. Rapport sur le service de transmission des correspondances du bureau civil. Archives du CICR, PFF 18. 
et que vous aurez une réponse à transmettre à ce comité» (lettre du 8 septembre 1917).

Dans la première lettre conservée, Ferrière s'exprime d'abord en médecin; il se préoccupe de son état de santé et le conseille:

Soyez très prudent notre climat extra humide est traître; ne sortez pas avant que vous vous sentiez nettement en bonne voie (lettre du 23 octobre 1914).

Et il n'a pas tort, car à peine un mois s'écoule que Rolland attrape une grippe et ne peut se rendre à l'Agence. A Genève, Rolland semble aussi se heurter à des résistances de toutes sortes qui déjà le rapprochent de Ferrière:

Depuis trois mois que je suis à Genève, j'ai appris non seulement à admirer et à aimer votre œuvre et votre cœur, mais à sentir la rareté, même dans ce milieu que je croyais très libre, de vos idées, qui sont aussi les miennes (lettre du $1^{\text {er }}$ janvier 1915) ${ }^{30}$.

Cette proximité des idées fonde leur amitié. L’un écrivant: «Je me réjouis que le hasard (aidé, un peu, je crois, par votre volonté) m’ait appelé à collaborer, bien modestement, à votre tâche de l'Agence des prisonniers et à devenir ainsi votre ami, je l'espère du moins" (lettre du $1^{\text {er }}$ janvier 1915) et le second confirmant:

Il y a longtemps que votre besoin de sincérité malgré tout, sans parler de votre talent m'avait et nous avait en famille attiré à vous; vous avez trouvé en nous ce même besoin et c'est un point de contact plus précieux que beaucoup d'autres: merci donc de votre amitié et de votre bienveillante collaboration (lettre de 2 janvier 1915).

Début juillet, Romain quitte l'Agence en remettant à Ferrière un volume de Jean-Christophe et une lettre concentrant ses sentiments à son égard:

Au moment où je prends congé (momentanément) de l'agence des Prisonniers de guerre, je voudrais vous dire combien j'ai été heureux de vous connaître et de travailler auprès de vous depuis neuf mois. Certes, ma collaboration vous a été d'une aide bien médiocre; et d'autre part, nous n'avons jamais pu échanger, au milieu du travail, que des entretiens hâtifs. Mais ces petites causeries journalières m'étaient

30. Lettre retranscrite par Marc Reinhardt. 
bienfaisantes, en me faisant connaître votre large esprit de justice et d'humanité, à la fois sensible à toutes les iniquités et indulgent à toutes les faiblesses de cette pauvre espèce humaine, qui est plus absurde que méchante et qui est la première à souffrir du mal qu'elle fait: car il retombe fatalement sur elle. A une époque où presque tous les esprits sont entraînés par les passions de partis, même dans les pays neutres, cela m’a été un réconfort de trouver une pensée comme la vôtre, avec laquelle je me sentais toujours en pleine sympathie (lettre du 4 juillet 1915).

Et le 5 juillet 1915 Ferrière de lui répondre:

J'ai beaucoup joui de nos courts échanges à l'Agence à propos de mille détails journaliers; j'ai été parfois un peu honteux de vous avoir offert un interlocuteur d'une culture si médiocre, mais vous avez bien voulu ne jamais me le laisser sentir. Je suis heureux, de mon côté, d'avoir pu contribuer par une faible part à atténuer pour vous l'énorme tristesse de ce temps. En ce qui me concerne qu'il me suffise de vous dire que je risque de travailler avec moins de courage en voyant votre place vide à côté de moi. Vous m'avez plus aidé que vous ne le pensez et votre généreux bon sens m’a été d'un grand appui, aussi le mot «temporairement» que je trouve dans vos lignes ne me laisse-t-il pas indifférent, croyez-le bien, lors même que bien souvent je me suis dit que ce petit travail terre à terre était pour vous une généreuse perte de temps (lettre du 15 juillet 1915).

Le dévouement est réciproque entre ces deux hommes que dix-huit années séparent.

\section{Un soutien au-delà des frontières}

Le mercredi 21 juillet, Rolland part pour Thoune non sans confier encore une valise à Ferrière. Sa sœur le rejoindra alors que leur mère (qui n'aime pas la solitude) reste sous la bonne garde du médecin:

Si dans les premiers jours de notre absence et avant votre départ, vous voulez bien vous assurer une fois que ma mère supporte sans trop de peine sa solitude, vous me ferez le plus grand plaisir (lettre du 20 juillet 1915). 
L'attention prise par le couple Ferrière à la santé de $\mathrm{M}^{\mathrm{me}}$ Rolland lorsque son fils est absent renforce encore leur amitié. En partant, c'est encore à lui qu'elle laisse un pli qu'il soigne "dans un coffre-fort encastré dans le mur d'une de nos chambres" (lettre du 22 août 1915). Rolland n'a pas trouvé en Suisse allemande toute la tranquillité escomptée (les lettres concernant l'Agence le poursuivent), mais il y a rencontré Karl Spitteler ${ }^{31}$ :

Mon séjour en Suisse allemande m'a fait assez de bien; mais je n'y ai pas trouvé toute la tranquillité que j'aurais souhaitée. Les lettres vous poursuivent; les événements ne vous laissent pas beaucoup de trêve: ils me paraissent gros de menace (à l'intérieur, comme à l'extérieur). Je m'étonne toujours de la cécité des hommes politiques qui ne s'aperçoivent pas de ce qui se prépare, autour d'eux, auprès d'eux (lettre du 31 août 1915).

Puis, Rolland retrouve sa mère à Vevey avant de retourner à Genève:

Je viendrai sans doute vous redemander, en octobre, une petite place à votre table de l'Agence, où vous distribuez depuis un an aux malheureux de toutes les nations en guerre les paroles consolantes et l'espérance (lettre du 31 août 1915).

Et Ferrière de lui répondre:

«Mille merci de me faire espérer votre collaboration si dévouée pour l'automne, cela me et nous fait plus plaisir que vous ne pensez. Mais ce travail n'est-il pas bien monotone et terre à terre pour vous? (lettre du 2 septembre 1915).

Lorsqu'il revient à l'Agence en automne 1915, ce sont en effet à nouveau les préoccupations des lettres reçues qui constituent l'essentiel de leurs relations épistolaires: celles d'Elisabeth Rotten ${ }^{32}$, d'un soldat belge, d'un écrivain français, de Pottecher, d'un malheureux serbe, d'un officier

3I. Karl Spitteler (1845-1925) : écrivain et poète suisse allemand qui se distingua pendant la Première Guerre mondiale pour ses positions d'une Suisse neutre et désavouant la politique allemande; il reçut le Prix Nobel de littérature en 1919.

32. "Mademoiselle Dr Elisabeth Rotten, dont vous avez pu apprécier le grand cœur et la généreuse activité (Auskunft- und Hilfstelle für Deutsche im Ausland und Ausländer in Deutschland) m'écrit qu'elle aurait l'intention de venir parler de son œuvre, en Suisse, en petit comité» (lettre du 24 février 1917). 
français interné, d'un soldat français blessé, de son ami breton le peintre Julien Lemordant qui a fait le plafond du théâtre de Rennes devenu aveugle par une balle, etc. Mais ce sont aussi des questions de rapatriements et de mauvais traitements dans les camps allemands, de camps de représailles, de camps d'internements en Suisse, de la libération de trois cents pauvres gens, puis celles du renvoi des étudiants des deux camps pour leurs examens universitaires, et toujours diverses demandes d'internement en Suisse.

De retour de Vienne, Ferrière lui fait part de ses impressions de voyage dans une longue lettre du 26 septembre 1915, à laquelle Rolland ne répond que brièvement, pris par de nouvelles attaques et difficultés:

Excusez-moi de ne pas vous écrire plus longuement aujourd'hui. J'ai des lettres urgentes à envoyer. Je suis pas malheur assailli, ces jours-ci, d'une façon plus injurieuse et perfide que jamais; mon silence depuis deux mois n'a fait que redoubler l'animosité, - sans doute parce que ma pensée continue de cheminer dans le public et qu'on veut l'étouffer. La censure vient de refuser à mon éditeur même de prendre connaissance du manuscrit de mon recueil d'articles que je voulais mettre sous les yeux des lecteurs français afin qu'ils jugent de l'inanité des accusations. Telle est la liberté d'à présent (lettre du 29 septembre 1915).

Espérant qu'on lui rende justice, Ferrière va jusqu'à le comparer à Dreyfus:

Je suis désolé des ennuis que vous avez, mais je me souviens des angoisses de l'affaire Dreyfus, des calomnies qui ont alors accablé des hommes de hautes valeurs. Votre cause est plus noble encore, c'est celle de la dignité du [nom] français et de l'amour de l'humanité; je ne puis croire qu'on ne vous rende pleine justice - et mieux - dès que les passions ne seront plus seules à parler. Je n’aurais jamais cru que le bon sens pût pareillement être étouffé par l'agitation et cela si longuement. Mais au fond le public veut beaucoup moins savoir qu'éprouver (1 ${ }^{\text {er }}$ octobre 1915).

Leur amitié se renforce tout au long de leurs échanges et Rolland ne cesse de le rappeler. 


\section{Une amitié réconfortante}

"Amitié ", c'est le mot que Rolland emploie pour remercier Frédéric Ferrière de son soutien: «Merci de votre affectueuse lettre. Vous êtes très bon de m'avoir écrit ces lignes de sympathie. Je vous assure que votre amitié m'est précieuse et réconfortante» (lettre du 4 octobre 1915). C'est aussi avec le mot "réconfort» qu'il remercie Maya Ferrière en janvier 1916: «Vous savez tous l'affectueuse reconnaissance que j’ai pour votre famille, où j'ai trouvé le meilleur réconfort, depuis mes quinze mois de quasi-exil à Genève» (lettre du 29 janvier 1916).

En janvier 1916, Rolland est de retour à Genève et écrit depuis l'établissement thermal de Champel-les-Bains ${ }^{33}$. En été, il passe six jours à Sierre et trois à Montana (où il a comme guide le peintre Edmond Bille), mais sans rendre visite aux soldats internés. Puis il repart à Thoune (où il y a beaucoup d'internés) et où il «dégèle» de son hivernage à Montana. Dans cette lettre à Maya, où il lui fait part de sa rencontre avec son ami Julien Lemordant, il y réitère son affection pour ses parents:

Rappelez-moi, je vous prie, au bon souvenir de vos chers parents, auxquels je ne pense jamais sans une vraie tendresse. Je n'oublierai jamais toute la bonté qu'ils m’ont témoignée, ainsi qu'à ma mère, pendant ces années difficiles. Je remercie beaucoup votre père pour sa dernière réponse à ma demande, au sujet des deux prisonniers (lettre du 23 juin 1916).

De Thoune, il se soucie des siens et craint «les représailles»:

Combien je voudrais que les miens, fussent en Suisse! Je prévois, à bref délai, d'affreuses représailles pour l'affreux raid des avions sur Carlsruhe (lettre du 28 juin 1916).

Heureusement sa mère l'y rejoint, puis son père (qui a quatre-vingts ans) et sa sœur. Il demande à Frédéric Ferrière de ne pas donner son adresse (car les Français continuent de s'adresser à lui) :

Je sais pourquoi on s'adresse tant à moi, - sans doute à cause de mon livre qui se répand un peu partout (lettre du 14 juillet 1916).

33. A propos de cet établissement voir D. Ripoll (éd.), Champel-les-Bains. 
Et c'est jusqu'à son amitié qu'il donne qui serait dangereuse; il écrit à Maya Ferrière:

Mon ami Jouve est persécuté par l'élément officier (interné), à cause de son amitié pour moi (lettre du 14 juillet 1916).

Celles qui lient les deux familles sont ainsi un gage de grande confiance. A l'automne 1916, Rolland est à Sierre et ses parents sont retournés à Paris. Ses inquiétudes à leur sujet reprennent. Il écrit:

Il n'est pas gai d'être séparés des uns et des autres, - moins encore par la distance que par ces mesures vexatoires de censure postale et télégraphique, qui sont aussi vaines que tyranniques (lettre du 3 octobre 1916).

A propos du choix de ses quartiers d'hiver, il hésite à revenir à Genève:

Ce qui me fait hésiter à revenir à Genève, c'est qu'il m’y devient plus difficile de me défendre contre des amis et des ennemis également compromettant (Je vous parle en toute intimité). Je tiens essentiellement à garder mon indépendance de pensée et d'action (ibid.).

Mais il conclut:

Et je crois bien d'ailleurs que je n'échapperai pas à l'attraction de Champel (et de mes chers voisins) (ibid.).

Rolland doit se méfier de tous et Frédéric Ferrière garder une posture d'impartialité vis-à-vis des autorités genevoises et suisses. C'est pourquoi, tout en faisant partie du comité (avec Seippel et Rolland) qui distribuerait les bénéfices de la vente du livre de Gustave Dupin, il renonce à y inscrire son nom, ce que Romain Rolland comprend parfaitement:

Il est trop naturel que vous demandiez à ce que votre nom ne figure pas, tandis que vous travaillez à l'Agence: Dupin se reprocherait, plus que quiconque, de porter atteinte par une démarche imprudente à votre universel et légitime renom d'impartialité; trop de malheureux en ont besoin (lettre du 10 novembre 1916).

A Genève, Rolland reçoit de nouvelles lettres: 
Quant aux correspondants, qui m'écrivent à l'Agence, c'est sans doute que mon volume: $A u$-dessus de la mêlée leur y a révélé ma présence; et la plupart ne m'écrivent que pour me demander des renseignements au sujet de parents disparus" (lettre du 24 janvier 1917).

Tout en s'émouvant de leur contenu, Rolland craint aussi d'être trompé. Il demande alors des renseignements supplémentaires à Ferrière:

Pourriez-vous avoir quelques moyens de savoir ce qu'il en est exactement de ce garçon? S'il est vraiment aussi malade qu'il le dit, et dénué de ressources, je contribuerais volontiers à une part de sa pension, dans une clinique. Mais je crains d'être trompé. (Cela m’est arrivé plus d'une fois). Sans vous donner la peine de vous informer vous-même, pourriez-vous m'indiquer un docteur de Leysin, qui me renseignerait? (lettre du 7 juin 1918).

Il lui faut des certificats médicaux et aussi vérifier les informations notamment à la "commission médicale suisse", auprès de l'officier de recherche.

A chaque passage à Genève, il rend visite à Frédéric Ferrière ou à sa femme à qui il écrit: "Je vous ferai un peu de musique, si cela ne vous ennuie pas» (lettre du 28 mars 1917).

\section{Une amitié par-delà les frontières}

Dès avril 1917, Rolland habite à Villeneuve à l'hôtel Byron où sa famille cherche à le rejoindre. Mais en juin, la situation politique s'aggrave et il s'en inquiète:

J'aurais souhaité de pouvoir causer avec vous de vos impressions de voyage. J'entrevois, à travers les lignes de votre lettre, que la situation est grave en Autriche, Hongrie, Bulgarie. - Entre nous et tout à fait confidentiellement, elle est aussi très grave en France. (Je suis fort exactement renseigné). Mais il règne dans les sphères politiques - et la presse qui est à leurs gages - un étrange aveuglement, légèreté? méconnaissance des choses une sorte de politique de l'autruche, qui se cache la tête derrière une pierre, pour ne pas voir ce qui lui serait désagréable (lettre du 16 juin 1917). 
Plus aucun passeport (ou visa) n'est alors accordé de la France pour la Suisse; ces difficultés empêcheront sa sœur et son père de venir avant la fin du mois de juillet.

Difficulté encore et toujours avec les journalistes qui ne manquent pas de l'épingler sur le retard du versement du montant du Prix Nobel qu'il a reçu et veut remettre à l'Agence (50000 fr.). Il ne lui sera remis que le $1^{\text {er }}$ juin. C'est qu'il faut compter avec la presse et «le moindre petit article de journal fait plus d'impression sur les maitres de l'heure que les forces morales et sociales, qui agissent en silence" (lettre du 12 septembre 1917).

En juin 1917, Romain Rolland peut s'engager clairement auprès de Ferrière; il lui écrit:

Je vais recevoir, ces jours-ci le montant de mon prix Nobel. Je désire que le premier usage que j'en ferai soit au profit de cette Agence Internationale des Prisonniers de Genève dont j'ai pu apprécier, comme humble collaborateur, l'admirable dévouement (lettre du 10 juin 1917).

La réponse de Ferrière est claire:

Je vous proposerai d'adresser votre don au comité de la Croix-Rouge pour l'Agence internationale des prisonniers de guerre de Genève. $\mathrm{Si}$ vous voulez [...] mettre une partie de cette somme à la disposition de mon service civil, vous pourriez indiquer dans votre lettre: "don "tant" (une petite partie seulement) à la disposition du chef de la section civile de l'Agence», ou telle indication analogue (lettre du 11 septembre 1917).

En juillet, sa mère a pu passer la frontière, mais ni son père ni sa sœur : «Ils se heurtent à des difficultés de passeport. Chaque pays, à présent, est devenu une prison» (lettre du 11 juillet 1917). Ils partiront le 27 juillet pour Evian.

Fin septembre 1917, la famille retourne à Paris et Romain Rolland est à Sierre avec son ami Jouve sous un climat qui lui convient à cause de l'ensoleillement. Adolphe Ferrière l'invite alors dans son chalet aux Pléiades et accepte de recevoir aussi (comme semble lui demander Rolland) ses papiers, tout en le rendant attentif au fait que le chalet est en bois et qu'il peut brûler: "Voyez si vous voulez vous procurer un coffre à l'abri du feu ou si vous consentez à associer vos papiers aux miens 
dans le même risque.» Il ajoute: «Mes documents représentent vingt ans de travail." (lettre du 22 novembre 1917).

Après l'installation en octobre 1917 du régime bolchevique, une longue lettre d'un Frédéric Ferrière bien découragé par les difficultés qu'il rencontre pour les rapatriements et bien déçu par la politique adoptée par les révolutionnaires:

Je suis bien déçu des Lénine et des Trotski qui, à peine au pouvoir, renchérissent sur les tyrans qu'ils ont voulu abattre et parlent de guillotine; j'ai su, par des renseignements privés, que les massacres d'officiers ont été terribles en Russie, à Petrograd en particulier. C'est la ruée des [inférieurs] malmenés sur leurs chefs arrogants, conséquences fatale du [militarisme]. Triste perspectives de la revanche populaire: homo homini lupus! (lettre du 20 décembre 1917).

Rolland lui renvoyant ses espoirs pour la nouvelle année qui pourrait être celle de la fin de la guerre:

Malgré tout, je crois que l'année qui vient verra la fin de la guerre. Ce ne sera pas la faute des gouvernants si elle se termine avant l'extermination totale des gouvernés; mais les meilleures choses ont une fin et il me semble que les peuples commencent à se lasser de cette excellente plaisanterie (lettre du 27 décembre 1917).

Le 6 février 1918, le comité international de la Croix-Rouge, publie un "Appel aux belligérants» contre l'emploi de gaz vénéneux (gaz moutarde), d'usage contraire au règlement adopté à La Haye. Paru dans la presse, notamment dans Le journal de Genève, cet appel en fera réagir plus d'un, dont Jean Debrit rédacteur du journal (fils de Marc Debrit son directeur) estimant que la Croix-Rouge est sortie de son mandat. Rolland écrit alors à Ferrière:

Et c'est pourquoi j'en veux à Debrit d'avoir attaqué la Croix-Rouge, précisément pour un de ses actes les plus courageux et dont l'avenir lui saura le plus de gré. J'ai horreur de tous les fanatismes, de tous les doctrinaires, de droite et de gauche, de la paix comme de la guerre. Avant tout, être humain, et garder son bon sens - s'il est encore possible, en notre temps (lettre du 15 février 1918).

Cette polémique entre Rolland et Debrit les séparera: 
Mais l'attitude de Debrit dans cette question d'humanité m'a séparé de lui. La polémique l'aveugle. Il y perd tout sentiment de la mesure et de la justice (lettre du 21 février 1918).

Ferrière de même juge cette polémique inutile à leur cause car toujours outrancière:

La haine crée-t-elle jamais le progrès, élève-t-elle les nations? A cet égard, je répugne beaucoup aux méthodes de polémique de Debrit, Guilbeaux et autres qui se meuvent dans une atmosphère de violence et prêchent la haine de classes ou de partis, au lieu de la combattre par des arguments faisant appel au bon sens et à la raison: faire réfléchir au lieu d'exciter les passions.

Et de proposer à Rolland un nouveau chapitre d'Au-dessus de la mêlée sur la folie des états en guerre (lettre du 23 février 1918).

En avril 1918, le chalet d'Adolphe Ferrière prend feu: il perd ses trois cent vingt cartons d'archives, trois mille livres et cinq manuscrits prêts à l'impression. Frédéric Ferrière qui y séjournait a dû sauter par la fenêtre; leur jeune bonne y trouve la mort par asphyxie ${ }^{34}$. Ce mois d'avril, la famille de Rolland se retrouve à Evian et la mère est dans l'impossibilité de passer la frontière en Suisse. Rolland lui écrit:

Je les ai vus tous trois hier, Dimanche, sur le pont de Gingolph; nous avons pu échanger 2 ou 3 mots; mais un gendarme est venu aussitôt nous enjoindre hautement de finir. - Je vous avoue que je ne suis pas fait pour subir un despotisme, de quelque couleur qu'il soit, impérial en républicain: je passe mon temps dans un état de révolte intérieure (lettre du 8 avril 1918).

Les contacts sont difficiles pour la famille Rolland «écartelée»: il faut trouver des passeurs pour les lettres ou se voir au pont de St-Gingolph à l'arrivée du bateau venant d'Evian. A chaque nouvelle ouverture de frontières, on s'y précipite:

34. Romain Rolland compatit: "Mais quel désastre! Sans parler de cette pauvre petite fille, que de douloureuses ruines pour votre fils Adolphe! Seul, un travailleur intellectuel, comme vous, peut se douter de la catastrophe que représente la disparition de tant d'années de notes, de recherches, d'écrits. Puisse ce coup si dur surexciter l'énergie de votre fils, au lieu de l'abattre! Il est assez jeune et assez fort pour reconstruire son œuvre plus large et plus complète qu'avant» (lettre du 3 avril 1918). 
Mon père et ma sœur nous sont arrivés brusquement. Ils ont profité de la brève ouverture de frontière, de lundi. Ils sont en bonne santé. Espérons qu'ils n'attraperont pas la grippe espagnole, qui est à Villeneuve, et dans toute la région (lettre du 10 juillet 1918).

De même on profite des passages pour rencontrer Zweig ou Spitteler.

\section{Un réseau de soutien}

Attaqué, "calomnié » ${ }^{35}$, en juillet 1918, par Diodore-Loyson et Isabelle Debran, Rolland reçoit une fois encore le soutien des Ferrière ${ }^{36}$. Seippel qui, dans un premier temps, ne cherche pas à démentir leurs allégations et refuse de publier une réaction de Rolland, se verra convaincre par Frédéric Ferrière de publier une note rectificative. De son côté Adolphe Ferrière écrit un article dans L'Essor. Sa réponse au pamphlet de Loyson entraîne dans les jours qui suivent une lettre de remerciement de Rolland. Sans conteste ce geste renforce encore leur amitié. Et de se retrouver tous à Villeneuve, fin août, avant de nouveaux départs toujours si inquiétants pour Rolland: Frédéric Ferrière part en voyage pour trois mois et ses parents retournent à Paris.

Les échanges épistolaires se développent alors entre Rolland et Adolphe Ferrière qui est devenu rédacteur du journal évangélique et pacifiste L'Essor, ce dont Rolland le félicite; comme de s'être réinstallé dans un nouveau chalet aux Pléiades. La guerre est finie, mais pour Rolland la lutte continue:

Il y a, de toute part, en Allemagne, de belles flambées d'idéalisme qui rappellent 48. Puisse-t-on ne pas les étouffer! - Je tâche ci et là, de les faire connaître en France par L'Humanité et Le Populaire. - Mais un démon funeste essaie de s'insinuer dans nos peuples éreintés - et peutêtre surtout chez les vainqueurs: - la lassitude, l'indifférence. Il faut lutter (lettre du 20 janvier 1919).

35. Titre de l'article de Charles Baudouin, «Romain Rolland calomnié. Réponse à une diffamation et analyse d'une méthode simple de suggestion collective».

36. Il s'agit du pamphlet publié à Genève (1918) par Isabelle Debran, préfacé par un certain Diodore et intitulé Monsieur Romain Rolland, initiateur du défaitisme. 
Cette nouvelle lutte, c'est autant contre l'impérialisme que contre les accusations personnelles:

L'impérialisme éhonté, la brutalité, le mensonge d'état (en particulier) s'étalent par toute l'Europe. Mais ce n'est pas une raison pour se laisser accabler (lettre du 12 mars 1919).

Il doit faire face autant à celle de traître à la patrie qu'à celle de compromission avec les Bolcheviques. Adolphe Ferrière devient aussi un confident. Rolland veut lui parler intimement, lui remettre des choses, lui fait part de l'attaque de sa mère qui l'a rendue aphasique. Plusieurs lettres de Rolland à Frédéric Ferrière détaillent l'état de santé de sa "chère maman" qui décède au mois de mai:

Nous avons beaucoup de peine à nous ressaisir. Ce coup nous a terrassés. Il y avait entre nous et notre chère maman une intimité si étroite qu'à tout instant elle nous manque, dans tout ce que nous faisons, dans tout ce que nous voyons, dans tout ce que nous pensons. J'ai perdu le meilleur de moi-même (lettre du 28 mai 1919).

Dans cette même lettre, il lui fait part depuis Paris de son projet d'acheter une maison en Savoie ou en Suisse pour y passer six mois par an (et y laisser ses livres et ses papiers qui sont toujours chez Frédéric et chez Adolphe) et c'est encore auprès d'eux qu'il cherche conseil:

Si vous ou les vôtres aviez un conseil à nous donner nous le recevrions avec plaisir. Les environs de Genève (savoisiens ou suisses) abondent de jolis coins; mais le climat est quelques fois un peu rude. Puisque $\mathrm{M}^{\text {lle }}$ Maya était, ces derniers temps, à Locarno, elle pourra dire ses impressions sur le pays, que je n'ai vu qu'en passant (lettre du 28 mai 1919).

De retour à Genève, en juillet, sa première visite est pour Frédéric Ferrière avant de repartir avec sa sœur à Schönbrunn fin août où leur père est en villégiature. A peine arrivé, il lui fait part sans réserve de sa position sociale qu'il semble bien partager avec les Ferrière:

L'époque est angoissante pour notre classe: elle se trouve étouffée entre les exigences ouvrières et les rapines des gros profiteurs. Je suppose que votre voyage récent a dû ajouter à vos soucis (lettre du 7 juillet 1919). 
Puis avant de repartir fin novembre pour Paris, il passe voir ses amis Ferrière et "hospitaliser" quelques papiers dans des malles en osier. De Paris il écrit alors à Maya Ferrière (Frédéric étant parti en voyage à Vienne) pour lui faire part de la grippe de son père et de sa propre bronchite:

On n'entend parler que de maladies, à Paris. L'affreux temps humide y contribue. - Il faut se bien défendre (lettre du 10 novembre 1919).

Après six ans de correspondances, de rencontres et d'amitiés, Romain Rolland fait tomber le mot "Monsieur»:

Cher ami (voulez-vous me permettre de supprimer le réfrigérant «Monsieur» et de vous prier de n'en plus faire usage avec moi?) (lettre du 30 mars 1920).

Nouvelle visite à Champel, nouveau dépôt de papier, nouvelle recherche de maison, nouvelle lettre à Frédéric Ferrière:

A la vérité, nous conduisons mon père à sa pension de Brunnen, et nous projetons de faire, cette année, au Tessin, le petit voyage de reconnaissance, (à la recherche d'une petite maison), - que nous n'avons pas eu le courage de faire, l'an dernier. Mais il va de soi que nous ferons aussi une halte, au bord du lac de Genève, pour voir nos chers amis Ferrière. J'espère bien que vous ne serez pas encore parti pour Vienne (lettre du 14 juin 1920).

Sans succès dans ses recherches immobilières, il retourne avec sa sœur à Paris.

\section{Une communauté d'idées et d'esprit}

En 1921, l'inquiétude de Rolland se porte sur la situation à Vienne, ce d'autant plus que Frédéric Ferrière y est; Romain et Madeleine voudraient par l'intermédiaire de la Société de secours aux enfants d'Europe, dont le siège est à Genève, s'engager à l'entretien d'un petit garçon de Vienne et d'une petite fille. Rolland est d'ailleurs "assiégé» de demande d'argent et sollicite à nouveau Ferrière pour vérifier ses sources.

En juin, de retour en Suisse, la première lettre de Rolland est pour le médecin. Mais ce dernier est alors malade, atteint d'un cancer à 
l'intestin, et Rolland fait le déplacement de Villeneuve à Genève pour le voir. Dans sa lettre il lui annonce qu'ils ont trouvé une petite maison dans le parc de l'Hôtel Byron:

Elle est jolie, et bien meublée. Nous y passerions un certain nombre de mois par année; et peut-être, à la fin de l'année prochaine, y ferais-je venir mes livres et meubles de Paris: car je ne sais encore si je pourrai renouveler, dans un an, mon bail qui va expirer à Paris. La crise du logement et la spéculation éhontée sont plus fortes que jamais. J'aime beaucoup ce pays de Villeneuve, qui est plein, pour moi, de souvenirs. Il me semble aussi qu'il doit être sain et bienfaisant (lettre du 6 juillet 1921).

Constamment Rolland s'intéressera à la santé du médecin, l'encourageant à espérer et à ne pas se décourager. Pour éviter une intervention chirurgicale, Ferrière subit des applications de radium (rayon X) qui sont très éprouvantes (congestion, inflammation). Rolland lui écrit:

Cher ami, je veux que vous sachiez bien avec quelle tendresse nous pensons constamment à vous et aux vôtres, en ces jours. Vous nous êtes bien plus proche que la plupart de nos parents. Tout ce qui vous atteint nous atteint. Nous avons l'esprit tendu vers votre guérison (lettre du 10 juillet 1921).

Et Ferrière de lui répondre:

J'ai plus de courage ces jours me sentant subjectivement un peu mieux, le jour que vous étiez ici j’ai été un peu lâche, pardonnez-le moi, je tiens tant à la vie et aux miens. Reportez plus tard sur eux l'affection que vous voulez bien me porter, ils la méritent (lettre du 13 juillet 1921).

Au début du mois de septembre avant de s'installer à l'autre bout du lac, Rolland passe quelques jours à Genève pour le voir. Mais pendant l'automne, c'est aussi Rolland qui est malade et plusieurs lettres à Ferrière décrivent par le menu ce mal qu'il avait ressenti jadis à vingt ans, lié à la tuberculose.

Leurs lettres se montrent de plus en plus affectueuses, espérant secrètement que l'affection des amis puisse avoir quelque influence sur la santé des uns et des autres. Rolland écrit depuis Paris: «Bon courage et espoir, ami que nous aimons!» (lettre du 29 novembre 1921). Et Ferrière de lui répondre: 
Hélas nous n'en sommes pas encore au radium de l'esprit; si la sympathie, l'ardent désir de voir les siens et ceux qu'on aime protégés, pourraient être efficaces, nous n'aurions pas assisté aux désespoirs sans nom, désespoirs auxquels on ne peut songer sans frémir, qui ont sévi sur l'Europe depuis 1914 (lettre du 29 décembre 1921).

Rolland déménage depuis Paris et s'installe à Villeneuve, s'excusant de n'avoir pas écrit en ce début d'année 1922. Le jeudi 29 juin, il vient à Genève pour rendre visite à Ferrière assommé de calmant pour supporter la douleur:

Excusez encore le piètre accueil d'un pauvre malade qui sent ses ressources intellectuelles et physiques se tarir, mille merci pour votre bonne affection que je mérite peu et croyez-moi, cher ami, votre bien sincèrement dévoué, Dr. Ferrière (lettre du $1^{\text {er }}$ juillet 1922).

Les deux amis se verront une dernière fois en août. Moins d'échange épistolaire entre eux: une lettre de Ferrière le 21 août, une de Rolland le 31 octobre, dans laquelle il lui fait part de son excommunication:

J'ai eu les honneurs d'une double excommunication, dans L'Humanité et dans Clarté, de la main du généralissime en personne, Trotsky. Violences rouges, violences noires, le lapin n’a pas de préférences sur la sauce à laquelle il doit être mangé. Mais moi, je ne veux pas être mangé du tout. Et on n'aura pas ma peau! (Vous voyez que je parle dans leur aimable langage) (lettre du 31 octobre 1922).

Pour la nouvelle année, Rolland lui écrit:

Nous vous envoyons, ainsi qu'aux chers vôtres, nos vœux les plus affectueux, pour que l'année qui vient vous apporte la guérison. Elle ne peut faire de plus beau présent à tous ceux qui vous aiment; et vous savez que nous sommes du nombre (lettre du 29 décembre 1922).

En 1923, Romain Rolland a envoyé en France un "Appel " pour venir en aide aux malheureux d'Allemagne qui en fera réagir plus d'un ${ }^{37}$. Longue lettre encore de Rolland fin décembre. Alors qu'il se plonge dans Gandhi pour ses articles dans la revue Europe, Ferrière souffre et tente par deux

37. Notamment: Anatole France, Paul Desjardins, Roger Martin du Gard, Charles Gide. 
fois des applications de radium: "Je tiens à la vie. Je désire vivre je me sens encore de la jeunesse en moi et la mort me révolte" (lettre du 6 avril 1923). Dernière lettre de Ferrière conservée.

Dernière visite de Rolland le 21 juin 1923 et deux dernières lettres de Rolland au médecin le 29 décembre 1923 et le 7 mai 1924:

Pardonnez-moi de ne vous avoir pas écrit depuis longtemps. On voudrait plutôt causer, quand on ne s'est pas vu depuis des mois: car on a beaucoup à dire, et peu à raconter. - Vous savez d'ailleurs que je me débats toujours au milieu des mille et une correspondances; je vais enfin me décider à prendre une secrétaire, un jour par semaine (pour commencer) : c'est devenu indispensable; je n'ai plus le temps de travailler pour moi (lettre du 7 mai 1924).

Puis Rolland prend le train pour Vienne et Prague, voyage d'un mois où il rencontre bien des amis de Ferrière. Dans une lettre du 12 juin, de retour à Villeneuve, il espère le revoir, mais Frédéric Ferrière meurt le samedi 14 juin, à 76 ans; une mort dont il dit qu'il ne se consolera jamais (lettre du 23 juin 1924 ${ }^{38}$ ). Il écrit dans son journal "Samedi 14 juin 1924. Mon cher Docteur Frédéric Ferrière est mort» et à la famille Ferrière:

Je vous apporte mon chagrin, et je le joins au vôtre. Depuis la mort de ma mère, c'est le plus douloureux que j'ai ressenti. Vous ne pouvez savoir, et le bon docteur lui-même sans doute ne l'a pas su - (je le lui ai mal montré) - l'affection que j'avais pour lui. Il m'était beaucoup plus qu'un ami. Depuis presque dix ans - (dix ans, au mois d'octobre) - que je l'avais connu, je me sentais lié à lui par l'attachement et la vénération. Aucun homme ne m’a inspiré un tel sentiment de respect affectueux. Jamais je n'oublierai ce qu'il a été pour moi, pendant les années de guerre. Ce n'était pas seulement la bonté qu'il nous a montrée personnellement, à ma mère et à moi. C'était sa bonté même, sa bonté en toute occasion, son dévouement incessant, son oubli de soi-même, son absolu, entier, perpétuel désintéressement. Il était si modeste qu'il ne s'est jamais douté de sa grandeur morale - et presque, de sa sainteté. Il eût été blessé, comme d'une fausse note, si on le lui avait dit. Je le disais, dans mon cœur. Puisse-t-il l'entendre, aujourd'hui! Ses peines sont finies. Nous restons avec les nôtres. Mais sa pensée nous accompagne. Jusqu’à mon dernier jour, je ne cesserai de

38. Lettre retranscrite par Sven Stelling Michaud. 
servir sa présence invisible et de lui demander un appui. Chers amis, permettez-moi de vous embrasser, comme un de votre famille, et de vous dire notre tristesse et notre affection profondes, de ma sœur et de moi. Mon père se joint à nous. Respectueusement Romain Rolland ${ }^{39}$.

A partir de juin 1924, la correspondance se poursuit avec les descendants du médecin Frédéric Ferrière et en particulier avec Adolphe cultivant désormais le souvenir de cette amitié:

Mon père a donné à Maya vos Jean-Christophe si magnifiquement reliés et vos autres ouvrages. Vous savez combien il aimait vos livres. C'étaient des trésors pour lui. Il était si fier de votre amitié! Elle a été l'une des grandes consolations de sa vie, si pauvre en satisfactions et en reconnaissance - dans le double sens du terme - venues du dehors (lettre du 19 juin 1924).

Rappelant encore dans une lettre de 1932 (après la mort d'Adolphine Ferrière-Faber) la place que Rolland avait occupée auprès de ses parents:

Maintenant nous contemplons mieux leur double action harmonisée, action sur nous, sur le reste de la famille et auprès des amis, parmi lesquels, dès le premier contact, vous aviez conquis le premier rang. Votre confiance, votre attachement si simple et si fidèle ont constitué une source de force pour nos parents. Penser à vous, à votre rectitude impitoyable, à votre courage, leur était un réconfort. Et c'est pourquoi, pour tout ce passé qui continue à vivre, nous vous disons un "merci» du fond du cœur! (lettre du 6 février 1932).

\section{La petite communauté des âmes en paix}

Ces échanges entre Frédéric Ferrière et Romain Rolland éclairent leur commune posture vis-à-vis de la guerre et plus largement du monde.

39. Les termes de cette lettre sont repris dans son journal où il décrit plus précisément l'affection qu'il lui portait: «Nul ne pourra savoir (et lui-même ne l'a pas su) l'affection et la vénération que je lui avais vouées. Je le connaissais depuis 10 ans. Ce qu'il a été pour moi (et pour ma mère) en ces années de guerre, moi seul (et ma mère) avons pu le sentir. Beaucoup plus qu'un ami. Quelqu'un de ma famille, - plus proche que mon père, - une âme de mon sang, mais plus pure, plus sainte.» Extrait du Journal intime de Romain Rolland. 
Leur correspondance peut être lue comme une manière de s'épauler l'un et l'autre pour tenir bon (à côté de leur travail respectif) dans un univers où ils sont bien peu à incarner une position politique et philosophique humaniste et universaliste. Autour d'eux quelques noms comme Georg Friedrich Nicolai ${ }^{40}$, Jouve, font partie de cette "petite communauté des âmes en paix avec le monde entier " comme l'écrit Romain Rolland (lettre du 7 août 1921).

Par leur correspondance, on comprend mieux le fonctionnement de la section civile des prisonniers de guerre, avec le type de demandes faites, les échanges entre les "civilistes», qui expliquent en partie d'ailleurs cette correspondance, et plus généralement le rôle de la Croix-Rouge internationale: recherche des prisonniers militaires et civils, amélioration de leur sort dans les camps d'internement et négociation des conditions de rapatriement. C'est aussi, évidemment, le rôle de la Suisse qui est souligné non seulement par l'activité de ces pacifistes et de l'Agence, mais aussi dans l'internement des prisonniers étrangers en Suisse, page encore peu étudiée de l'histoire suisse ${ }^{41}$.

In fine, cette correspondance - archive de la vie privée - est un voyage dans l'intimité de ces grandes âmes que sont les deux protagonistes, lesquels grâce à leur amitié nous confie leur combat au quotidien pour leur conception commune d'une société où l'être humain serait premier.

\author{
Martine RUCHAT \\ Université de Genève
}

\footnotetext{
40. Georg Friedrich Nicolaï (1874-1964) : médecin et professeur de physiologie et de médecine à l'Université de Berlin, s'étant opposé à la guerre, il fut arrêté et emprisonné. Son ouvrage Die Biologie des Krieges (La Biologie de la Guerre) est interdit en Allemagne et sera publié en 1917 à Zurich.

4I. Voir D. Michelian, L'internement des réfugiés en Valais pendant la seconde guerre mondiale.
} 


\section{BIBLIOGRAPHIE}

\section{Sources}

Baudouin, Charles, "Romain Rolland calomnié. Réponse à une diffamation et analyse d'une méthode simple de suggestion collective», Genève, Cahiers du Carmel, 1918.

Extraits de lettres d'Adolphe et Maya Ferrière à Romain Rolland, Bibliothèque nationale de France, fonds Romain Rolland.

Extraits de lettres de Romain Rolland à Frédéric Ferrière, Archives d'Etat de Genève, Archives privées 379.

Extrait du Journal intime de Romain Rolland (1 janvier- 6 décembre 1924), Archives d'Etat de Genève, Archives privées 379.

\section{Travaux}

Abraham, Pierre, Barrère, Jean-Bertrand, Buenzod, Janine, Chavanne, André, Reinhardt, Marc, Stelling Michaud, Sven, Romain Rolland, suivi de la correspondance inédite de Romain Rolland avec Adolphe Ferrière et Heinz Häberlin, Neuchâtel, A la Baconnière, 1969.

Armanios, Rachad, Le Dr. Ferrière: les années de formation d'un médecin et d'un philanthrope, mémoire de licence, Genève, 2003.

Basquin, Claire, Romain Roland et l'Agence des prisonniers de Genève (1914-1916), thèse pour le diplôme d'archiviste paléographe, Paris, Ecole des Chartes, 1999.

Buum, Antoinette (éd.), Correspondance entre Romain Rolland et Charles Baudouin: une si fidèle amitié: choix de lettres (1916-1944), avantpropos d'Yves Baudouin, Meyzieu, Césura, 2000.

Duchatelet, Bernard, Romain Rolland tel qu'en lui-même, Paris, Albin Michel, 2002. 
Ferrière, Adolphe, Le Dr. Frédéric Ferrière - Son action à la CroixRouge internationale en faveur des civils victimes de la guerre, Genève, Suzerenne, 1948.

L'agence internationale des prisonniers de guerre. Le CICR dans la première guerre mondiale, Genève, Musée international de la Croix rouge et du croissant rouge, 2007.

Michelian, David, L'internement des réfugiés en Valais pendant la seconde guerre mondiale: entre univers laborieux et sociabilité, mémoire de licence, Fribourg, 2006.

Reinhardt, Marc, «Romain Rolland et les Ferrière. Visage d'une correspondance», Versants, 7 (1985), p. 137-160.

Ripoll, David (éd.), Champel-les-Bains, Gollion, Infolio, 2012.

Werner, Georges, "Frédéric Ferrière 1848-1924", Revue internationale de la Croix-Rouge, 67 (16 juillet 1924), p. 485-543. 
